\title{
Computer Simulation of Continuous Casting Processes: A Review
}

\author{
Nitin Amratav, Kulyant Kumar, Megad Pillai
}

Department of Metallurgy, College of Engineering, Amravati, India

Email address:

niti.amratava@gmail.com (N. Amratav)

\section{To cite this article:}

Nitin Amratav, Kulyant Kumar, Megad Pillai. Computer Simulation of Continuous Casting Processes: A Review. Advances in Materials. Vol. 10, No. 3, 2021, pp. 31-41. doi: 10.11648/j.am.20211003.11

Received: August 28, 2021; Accepted: September 13, 2021; Published: September 29, 2021

\begin{abstract}
Steelmaking is the second step in producing steel from iron ore. In this stage, impurities such as sulfur, phosphorus, and excess carbon are removed from the raw iron, and alloying elements such as manganese, nickel, chromium, and vanadium are added to produce the exact steel required. Modern steelmaking processes are broken into two categories: primary and secondary steelmaking. Primary steelmaking uses mostly new iron as the feedstock, usually from a blast furnace. Secondary steelmaking uses scrap steel as the primary raw material. Gases created during the production of steel can be used as a power source. Steelmaking is presently a grounded innovation driven by plant, exploratory and computational examination. The continuous casting process comprises many complicated phenomena in terms of fluid flow, heat transfer, and structural deformation. The important numerical modeling method of the continuous casting process has been discussed in reference in this work. With the recent advancement in metallurgical methods, the continuous casting process now becomes the main method for steel production. To achieve efficient and effective production, the manufacturers of steel keep on searching for new methods which increase productivity. The present work describes molten steel flow, heat transfer, solidification, electromagnetic applications, formation of the shell by solidification and coupling, etc.
\end{abstract}

Keywords: Steelmaking, Metallurgy, Computer Simulation, Continuous Casting

\section{Introduction}

Steelmaking is presently a grounded innovation driven by plant, exploratory and computational examination. The purpose for this is the benefits that accompany the nonstop projecting cycle which incorporates cost-saving, high efficiency, and better quality [1-6]. To achieve efficient and effective production, the manufacturers of steel keep on searching for new methods which increase productivity. One such kind of method has become more popular to use optimizing using numerical modeling. The continuous casting process comprises many complicated phenomena in terms of fluid flow, heat transfer and structural deformation [7-17]. The important part and process of continuous casting have been modeled in-depth and discussed in reference [18]. It describes molten steel flow, formation of the shell by solidification. Further, the distortion of strand by thermomechanical forces, bulging, bending and crack prediction has been also given in detail. Till now, many powerful pre-coded solvers are available in the market. The numerical simulation of the thermo-mechanical behavior of the continuous casting process is important in terms of achieving a quality product [19-26].

This part of simulation comes with many obstacles such as dealing with the highly non-linear constitutive laws of structure, incorporation of latent heat, involvement of three different states of material: liquid, mushy and solid, temperature-dependent material properties, irregular contact between the mold surface and solidified strand, and coupling the heat transfer and structure model with proper continuum mechanism and boundary condition [27]. Reynold's Averaged Navier- Stokes (RANS) method has been widely adopted for turbulence modeling. It has been reported that the RANS model is highly accurate in predicting steady-state flow patterns [23]. The research work done in the last three decades has made continuous casting an advanced and sophisticated technology [18, 28-30]. Physical water models can simulate the molten steel flow in the mold region of the continuous casting process considering the viscosity of water equivalent to steel [31-35]. Several research works have been 
done on molten steel flow, heat transfer and solidification in mold [33-38]. These studies have been established and validated with industrial trials [7, 28, 39-42]. From all previous studies, it is well established that numerical models efficiently and accurately predict the fluid flow and mechanical behavior of mold and strand, respectively [31, 32]. In this present work, we have reviewed the literature to provide current information on the mathematical modeling of steelmaking tundish.

\section{Computer Modeling of Mass Flow}

The molten steel flow in continuous casting mold is usually assumed to have some characteristics. These flow characteristics are classified based on some assumptions such as compressible and incompressible. The molten steel flow is governed by the continuity equation and momentum equation, supplemented by heat transfer boundary conditions [43-45]. The governing equations related to mass flow and momentum transfer are as follows [46];

$$
\begin{aligned}
\nabla \cdot u & =0 \\
\rho\left(\frac{\partial u}{\partial t}+(u \cdot \nabla) u\right) & =f-\nabla p+\mu \nabla^{2} u
\end{aligned}
$$

In 2005, Zhao et al. [47] studied the transient molten steel flow and superheat transport in a continuous casting mold. The equation for momentum calculation was used as follows.

$$
\begin{aligned}
\rho_{0}\left(\frac{\partial \bar{u}_{i}}{\partial t}+\frac{\partial\left(\bar{u}_{i} \bar{u}_{j}\right)}{\partial x_{j}}\right)= & -\frac{\partial \bar{p}}{\partial x_{j}}+\frac{\partial}{\partial x_{j}}\left(\mu \frac{\partial \bar{u}_{i}}{\partial x_{j}}\right)+\frac{\partial Q_{i j}}{\partial x_{j}} \\
& +\delta_{i 3} \rho_{0} \beta\left(\bar{T}-T_{0}\right) g
\end{aligned}
$$

Further momentum equation was modified by using a subgrid momentum flux term $Q_{i j}$.

where,

$$
Q_{i j}=\rho_{0}\left(\bar{u}_{i} \bar{u}_{j}-\bar{u}_{i} \bar{u}_{j}\right) \text { Turbulence modeling }
$$

Most of the previous work on continuous casting mold has been modeled using the RANS equation [47]. Therefore, turbulent viscosity was predicted for the Large Eddy Simulation (LES) model from the following equation.

$$
\mu_{T}=C_{v} \rho_{0} K_{G}^{1 / 2} \Delta
$$

where the constant $C_{v}$ is 0.05 , and $\Delta$ is the grid-length scale, given by $\Delta=\left(\Delta_{x} \Delta_{y} \Delta_{z}\right)^{1 / 3}\left(\Delta_{x}, \Delta_{y}\right.$, and $\Delta_{z}$ are grid sizes in the $x, y$, and $z$ directions, respectively).

To understand the complex flow profile in mold, $\mathrm{Li}$ and Tsukihashi [48] have developed a numerical model to investigate the vortexing flow in SEN of continuous casting of steel. To describe the behavior of vortices in the flow field, definitions of the vorticity $\omega_{z}$ was expressed as follows,

$$
\omega_{z}=\frac{1}{2}\left(\frac{\partial u_{y}}{\partial x}-\frac{\partial u_{x}}{\partial \theta}\right)
$$

Sowa and Bokota [49] have assumed viscous incompressible and laminar flow to describes flow patterns in mold. They proposed the following equation for mass and momentum calculation.

$$
\begin{gathered}
\nabla \cdot v=0 \\
\rho \frac{d \mathrm{v}}{d t}=\rho \mathrm{g}-\nabla p+\mu \nabla^{2} \mathrm{v}
\end{gathered}
$$

In a similar work in 2013, Zare et al. [50] investigated the molten steel flow filed in the mold under various conditions of submerged entry nozzle. In their work, the following momentum equation was solved;

$$
\frac{\partial\left(\rho v_{i} v_{j}\right)}{\partial X_{i}}=-\frac{\partial p}{\partial X_{i}}+\frac{\partial}{\partial X_{i}}\left[\mu_{e f f}\left(\frac{\partial v_{i}}{\partial X_{i}}+\frac{\partial v_{i}}{\partial X_{j}}\right)\right]+\rho g_{j}+F_{j}
$$

In the above equation, Zare et al. (2013) [50] used the effective viscosity term in the momentum equation. The terms $\mathrm{k}$ and $\varepsilon$ for turbulent viscosity were predicted from two equations of the standard $\mathrm{k}-\varepsilon$ model. It was expressed as follows.

$$
\mu_{e f f}=\mu+\mu_{t}
$$

$\mu_{t}$ can be calculated using $k-\varepsilon$ parameters:

$$
\mu_{t}=C_{\mu} \rho \frac{k^{2}}{\varepsilon}
$$

The modified equation for momentum was given as follows [44];

$$
\begin{array}{r}
\frac{\partial}{\partial t}(\rho u)+\nabla \cdot(\rho u u)=-\nabla p+\nabla \cdot\left[\left(\mu_{\ell}+\mu_{t}\right) \nabla u\right]+\rho g+ \\
\rho g \beta\left(T-T_{0}\right)+\frac{\left(1-f_{\text {liq }}\right)^{2}}{f_{\text {liq }}^{3}+0.001} A_{\text {mush }}\left(u-u_{\mathrm{s}}\right)+F_{\text {ave }}(12)
\end{array}
$$

In the above equation, $f_{\text {liq }}$ the liquid fraction. The lever rule of solidification was utilized to calculate the mushy zone as follows.

$$
f_{\text {liq }}=1-\frac{1}{1-k_{0}} \frac{T-T_{\text {liq }}}{T-T_{\text {melt }}}
$$

One such popular model that works on the abovementioned method is the $\mathrm{k}-\varepsilon$ model Further, more details on the mathematical modeling of multi-phase fluid flow can be read elsewhere [20, 51-53].

Comparative studies have been carried out by many researchers to investigate the influence of various turbulence models on the estimation of results [54-59]. Siddiqui et al., [60] compared different turbulence models and predicted results revealed that the $\mathrm{RNG} k-\varepsilon$ model has a good approximation. The equations of motion for $k$ phase in an Euler-Euler simulation are generally given as follows:

$$
\begin{gathered}
\frac{\partial\left(\alpha_{k} \rho_{k}\right)}{\partial t}+\nabla \cdot\left(\alpha_{k} \rho_{k} u_{k}\right)=0 \\
\frac{\partial\left(\alpha_{k} \rho_{k} u_{k}\right)}{\partial t}+\nabla \cdot\left(\alpha_{k} \rho_{k} u_{k} u_{k}\right) \\
=-\nabla \cdot\left(\alpha_{k} \tau_{k}\right)-\alpha_{k} \nabla P+\alpha_{k} \rho_{k} g^{\prime}+M_{l, k}
\end{gathered}
$$

The stress terminology of $\mathrm{k}$ phase can be written as:

$$
\tau_{k}=-\mu_{e f f, k}\left(\nabla u_{k}+\left(\nabla u_{k}\right)^{T}-\frac{2}{3} \operatorname{I}\left(\nabla \cdot u_{k}\right)\right)
$$




$$
\mu_{e f f}=\mu_{L, I}+\mu_{T, 1}+\mu_{B l, 1}
$$

Empirically the calculation of effective viscosity of gas was calculated from effective liquid velocity.

$$
\mu_{e f, g}=\frac{\rho_{g}}{\rho_{l}} \mu_{e f f, l}
$$

The model proposed by Sato \& Sekiguchi ${ }^{22}$ has been used to take account of the turbulence induced by the movement of the bubbles. The expression is:

$$
\begin{gathered}
\mu_{B I, l}=\rho_{i} C_{\mu, B I} \alpha_{g} d_{g}\left|u_{g}-u_{i}\right| \\
u_{k}=\tilde{u}_{k}-u_{k}^{\prime}
\end{gathered}
$$

A numerical model has been developed to analyze the transient three-dimensional and three-phase flow in a bottom stirring ladle with a centered porous plug, which takes into account the steel, gas, and slag phases; it enables us to predict the fluid flow and heat transfer in the very important steel/slag region. They applied k- $\varepsilon$ turbulence model [61];

$$
\begin{array}{r}
\frac{\partial(\rho k)}{\partial t}+\rho u_{j} \frac{\partial k}{\partial x_{j}}=\frac{\partial}{\partial x_{j}}\left(\frac{\mu_{\mathrm{eff}}}{\sigma_{k}} \cdot \frac{\partial k}{\partial x_{j}}\right)+G_{k}+G_{b}-\rho \varepsilon \\
\frac{\partial(\rho \varepsilon)}{\partial t}+\rho u_{j} \frac{\partial \varepsilon}{\partial x_{j}}=\frac{\partial}{\partial x_{j}}\left(\frac{\mu_{\mathrm{eff}}}{\sigma_{\varepsilon}} \cdot \frac{\partial \varepsilon}{\partial x_{j}}\right)+\frac{\left(c_{1} G_{k} \varepsilon+c_{2} G_{b}-c_{3} \rho \varepsilon^{2}\right)}{k}
\end{array}
$$

In the above relationship, $\mathrm{G}_{\mathrm{k}}$ is the turbulent kinetic energy generated by mean flow velocity gradients. This can be written as follows;

$$
\begin{gathered}
G_{k}=\mu_{t} \frac{\partial u_{j}}{\partial x_{i}}\left(\frac{\partial u_{i}}{\partial x_{j}}+\frac{\partial u_{j}}{\partial x_{i}}\right) \\
G_{k}=\mu_{t} \frac{\partial u_{j}}{\partial x_{i}}\left(\frac{\partial u_{i}}{\partial x_{j}}+\frac{\partial u_{j}}{\partial x_{i}}\right)
\end{gathered}
$$

Further, $\mathrm{G}_{\mathrm{b}}$ shows the turbulent kinetic energy generated by buoyancy and it can be expressed as;

$$
G_{b}=-g \frac{\mu_{t}}{\rho \operatorname{Pr}_{t}} \frac{\partial \rho}{\partial x_{i}}
$$

The effective viscosity can be written as the addition of laminar and turbulent viscosities, as follows.

$$
\mu_{\mathrm{eff}}=\mu+\mu_{t}=\mu+\rho c_{\mu} \frac{k^{2}}{\varepsilon}
$$

The values for the constants in this $k-\varepsilon$ model $c_{1}, c_{2}, c_{3}$, $c_{u}, \sigma_{k}$, and $\sigma_{\varepsilon}$ are $1.43,1.92,0.09,1.00$, and 1.30 , respectively [15].

In 2014, Li et al. [62] developed a mathematical model to study the vortex formation in ladles. It is formed during liquid steel teeming from the ladle. They studied vortex formation during ladle teeming using new technology. The results obtained help to verify the validity of the numerical computations. [62]

Turbulent kinetic energy equation $(k)$ :

$$
\begin{gathered}
\frac{\partial}{\partial t}(\rho k)+\frac{\partial}{\partial x_{i}}\left(\rho k u_{i}\right)=\frac{\partial}{\partial x_{j}}\left(\alpha_{k} \mu_{e f f} \frac{\partial k}{\partial x_{j}}\right)+G_{k}+G_{b}-\rho \varepsilon- \\
Y_{M}+S_{k}
\end{gathered}
$$

Turbulent dissipation rate equation $(\varepsilon)$

$$
\begin{gathered}
\frac{\partial}{\partial t}(\rho \varepsilon)+\frac{\partial}{\partial x_{i}}\left(\rho \varepsilon u_{i}\right)=\frac{\partial}{\partial x_{j}}\left(\alpha_{\varepsilon} \mu_{e f f} \frac{\partial \varepsilon}{\partial x_{j}}\right)+C_{1 \varepsilon} \frac{\varepsilon}{k}\left(G_{k}+\right. \\
\left.C_{3 \varepsilon} G_{b}\right)-C_{2 \varepsilon} \rho \frac{\varepsilon^{2}}{k}-R_{\varepsilon}+S_{\varepsilon}
\end{gathered}
$$

In the past, various viscosity models have been used by the researchers to take care of the turbulence flow in the continuous casting process $[15,51,57,63-78]$.

\section{Equations for Heat Transfer and Solidification}

The fundamental requirement of the continuous casting process is to solidify the strand to achieve plant set quality standards [18, 30, 79-87] Generalized heat transfer equation (3-dimension) can be written in the most suitable format from the above equations in the following manner [46];

$$
\begin{gathered}
\rho C\left(\frac{\partial T}{\partial t}+V_{x} \frac{\partial T}{\partial t}+V_{y} \frac{\partial T}{\partial t}+V_{z} \frac{\partial T}{\partial t}\right)=\ddot{q}+\frac{\partial}{\partial x}\left(K_{x} \frac{\partial T}{\partial x}\right)+ \\
\frac{\partial}{\partial y}\left(K_{y} \frac{\partial T}{\partial y}\right)+\frac{\partial}{\partial z}\left(K_{z} \frac{\partial T}{\partial z}\right)
\end{gathered}
$$

In 2005, Louhenkilpi et al. proposed a three-dimensional transient formulation for temperature distribution over the mold wall. [88];

$$
\begin{gathered}
\rho \frac{\partial H}{\partial t}+v \frac{\partial H}{\partial z}=\frac{\partial}{\partial x}\left(k_{\mathrm{eff}} \frac{\partial T}{\partial x}\right)+\frac{\partial}{\partial y}\left(k_{\mathrm{eff}} \frac{\partial T}{\partial y}\right) \\
+\frac{\partial}{\partial z}\left(k_{\mathrm{eff}} \frac{\partial T}{\partial z}\right)
\end{gathered}
$$

In a similar work, Zhao et al. (2005) [47] modeled energy equation along with the Navier-Stokes equation.

$$
\begin{gathered}
\frac{\partial \bar{T}}{\partial t}+\frac{\partial\left(\bar{u}_{i} \bar{T}\right)}{\partial x_{i}}=\frac{k}{\rho_{0} C_{P}} \frac{\partial}{\partial x_{i}}\left(\frac{\partial \bar{T}}{\partial x_{i}}\right)+\frac{\partial Q_{T i}}{\partial x_{i}} \\
Q_{T i}=\bar{T} \bar{u}_{i}-\overline{T u}_{i} \\
\frac{\partial Q_{T i}}{\partial x_{i}}=\frac{\mu_{T}}{\operatorname{Pr}_{T}} \frac{\partial}{\partial x_{i}} \frac{\partial \bar{T}}{\partial x_{i}}
\end{gathered}
$$

In 2011, Sowa and Bokota [49] proposed a heat flow model based on the Fourier-Kirchhoff system of equations.

$$
\rho c\left(\frac{\partial T(\mathbf{x}, t)}{\partial t}+\nabla T \cdot \mathbf{v}\right)=\nabla \cdot(\lambda \nabla T)+\dot{Q}
$$

Sowa and Bakota et al. [49] modified the above equation which includes effective specific heat (Ceff) term which is a function of the temperature of the material.

$$
\begin{aligned}
& \nabla .(\lambda \Delta T)-C_{e f} \frac{\partial T}{\partial t}-C_{e f} \nabla T . V=0 \\
& C_{e f}(T)=\rho_{L S} c_{L S}+\rho_{S} L /\left(T_{L}-T_{S}\right)
\end{aligned}
$$

In 2011, Hadata et al., [37] proposed a steady FourierKirchhoff model for heat flow with some assumptions.

$$
q_{v}=Q_{s} \frac{d V_{s}}{d \tau}
$$


In a study in 1993 S. E. Chidiac et. at., [64] used enthalpy approach for heat transfer in multi-dimensional problem with following equation.

$$
\rho \frac{\partial H}{\partial t}=\nabla \cdot(K \nabla T)+Q
$$

where $\rho$ indicates density, $H$ indicates enthalpy, $K$ indicates Thermal conductivity, $Q$ indicates heat generation rate for unit volume, $T$ indicates temperature and $t$ time. Enthalpy is nothing but the summation of sensible $\&$ latent heat and can be expressed as:

$$
H=\int_{T_{r}}^{T} c d T+f(T) \cdot L
$$

where c, $f(T)$ and $L$ are specific heat liquid fraction and latent heat. For phase change study two methods are clubbed together with the above-stated formulation for accuracy and efficiency. Dirichlet \& Cauchy boundary conditions are used to solve above equations. The study carried in 2003, B. wiwanapataphee et. al., [63] for simulating phase change cause of heat transfer single domain enthalpy method is adopted. Where enthalpy is the summation of latent heat $(\mathrm{H})$ $\&$ sensible heat $(\mathrm{h})$.

$$
\mathrm{H}=\mathrm{h}+\Delta \mathrm{H}
$$

Latent heat $\mathrm{h}$ can be given by

$$
\mathrm{H}=f(T) L,
$$

Where L denoted Latent Heat of Steel $L$ and $f(T)$ indicates localized liquid fraction where value one represents complete Liquids state and zero represents the complete solid-state. The liquid fraction is nonlinear for simplification of the model it is assumed linear.

$$
f(T)= \begin{cases}0, & T \leqslant T_{s} \\ \frac{T-T_{S}}{T_{\mathrm{L}}-T_{\mathrm{S}}}, & T_{\mathrm{S}}<T<T_{\mathrm{L}} \\ 1, & T \geqslant T_{\mathrm{L}},(\mathrm{XX})\end{cases}
$$

Where in $T_{L}$ indicates melting temperature and $T_{S}$ Solidification temperature.

For region where phase change occurs conservation of energy principle. Combining this equation with enthalpy gives,

$$
\rho c\left(\frac{\partial T}{\partial t}+u_{j} T\right)=\left(k_{0} T_{j}\right)_{j}-S_{T}
$$

Ivanova (2013) [89] formulated extensive mathematical modeling on predicting phase-dependent boundary conditions.

$$
\begin{aligned}
& \frac{\partial T}{\partial r}+v(t) \cdot \frac{\partial T}{\partial z}=\frac{1}{c(T) \rho(T)} \times \\
& \times\left\{\frac{\partial}{\partial x}\left[\lambda(T) \frac{\partial T}{\partial x}\right]+\frac{\partial}{\partial \partial_{z}}\left[\lambda(T) \frac{\partial T}{\partial_{z}}\right]\right\}
\end{aligned}
$$

The position of the unknown phase boundary is specified by the equality condition of the temperatures and the Stefan condition for the two-dimensional case:

$$
\begin{array}{r}
T=\left.\left.T(\tau, x, z)\right|_{x=\xi_{-}(\tau, z)=} T(\tau, x, z)\right|_{x=\xi_{+}(\tau, z)=T_{c r}} \\
\lambda(T) \frac{\partial T}{\partial \bar{n}}\left|\xi_{+}-\lambda(T) \frac{\partial T}{\partial \bar{n}}\right| \xi_{-}=\mu \rho\left(T_{k p}\right)\left(\frac{d \xi}{d \tau}+v(\tau) \frac{d \xi}{d z}\right)
\end{array}
$$

where $\xi$ is the phase boundary $x=\xi(\tau, z), \bar{n}$ is a normal to the phase boundary, $\left.\frac{\partial T}{\partial \pi}\right|_{\xi+/-}$ is the left-right limit of the temperature derivative in the normal direction. $\mu$ is the latent the heat of crystallization. $T_{c r}$ is the crystallization temperature (the average temperature from the liquidussolidus interval).

In 2014, Zhang et al [90] investigated a steady-state twodimensional numerical model based on the assumption of heat transfer.

$$
\begin{gathered}
\rho=\left(1-f_{\mathrm{S}}\right) \rho_{\mathrm{L}}+f_{\mathrm{S}}\left(f_{\delta} \rho_{\delta}+f_{\gamma} \rho_{\gamma}\right) \\
\lambda=\left(1-f_{\mathrm{S}}\right) \lambda_{\mathrm{L}}+f_{\mathrm{S}}\left(f_{\delta} \lambda_{\delta}+f_{\gamma} \lambda_{\gamma}\right) \\
c_{\text {eff }}=f_{\mathrm{S}} \cdot c_{\mathrm{S}}+\left(1-f_{\mathrm{S}}\right) \cdot c_{\mathrm{L}}-L \frac{\partial f_{\mathrm{S}}}{\partial T}
\end{gathered}
$$

In a similar work, Maurya and Jha (2014) [91] investigated the effect of casting speed in the continuous casting process.

$$
\rho \frac{\partial H}{\partial t}+\rho \nabla \cdot(u H)=\nabla\left(k_{e f f} \nabla T\right)+Q_{\ell}
$$

Where $\rho$ is density, $H$ is enthalpy, $\Delta H$ is sensible heat, QL is source term. QL can be expressed as a single solidification model and given as;

$$
\begin{gathered}
Q_{L}=\rho L \frac{\partial f_{s}}{\partial t}+\rho L \bar{u}_{p u l l} \cdot \nabla f_{s} \\
\frac{\partial}{\partial x_{i}}\left(\rho u_{i}\right)=0
\end{gathered}
$$

Naiver-Stokes equation for transient momentum conservation is given by

$$
\frac{\partial}{\partial t}(\rho u)+\rho \nabla(u u)=-\nabla P+\nabla\left\{\mu_{e f f}(\nabla \cdot u)\right\}+\rho+S
$$

where,

$$
\mu_{\mathrm{eff}}=\mu_{1}+\mu_{\mathrm{t}}
$$

Maurya and Jha (2014) [91] and Hitanen et al. (2017) [92] used the enthalpy-porosity technique for solidification.

$$
S=\frac{(1-\beta)^{2}}{\left(\beta^{3}-\xi\right)} A_{\text {mush }}\left(\bar{u}-\bar{u}_{\text {pull }}\right)
$$

where, liquid fraction is expressed as $\beta, \xi=0.001$, mushy zone constant is given as $\mathrm{A}_{\text {mush }}$.

Pilvi et. at., (2017) [65] Used turbulent flow modelling at inlet in which they considered hydraulic diameter at inlet.

$$
\lambda_{e}=\lambda_{L} L\left(1+6\left(1-f_{s}\right)^{2}\right.
$$

In 2016, Hibbeler et al. [93] proposed an innovative reduced-order model (ROM) for heat transfer from mold in the continuous casting of steel. 


$$
0=\frac{\partial^{2} \theta_{\text {mould }}}{\partial x^{*} 2}+\left(\frac{d_{\text {mould }}}{w_{\text {mould }}}\right)^{2} \frac{\partial^{2} \theta_{\text {mould }}}{\partial y^{* 2}}+\left(\frac{d_{\text {mould }}}{\ell_{\text {mould }}}\right)^{2} \frac{\partial^{2} \theta_{\text {mould }}}{\partial z^{* 2}}
$$

Vnnyscy and Saleem (2017) [94] formulated a mathematical formulation for explicitly calculating the geometrical range of the mushy zone.

$$
\rho c_{\mathrm{p}} V_{\text {cast }} \frac{\partial T}{\partial z}=\frac{\partial}{\partial y}\left(k \frac{\partial T}{\partial y}\right)+\frac{\partial}{\partial z}\left(k \frac{\partial T}{\partial z}\right)-\rho V_{\text {cast }} \Delta H_{\mathrm{f}} \frac{\partial \chi}{\partial z}(56)
$$

where

$$
\begin{gathered}
k=\chi k_{1}+(1-\chi) k_{s} \\
c_{\mathrm{p}}=\chi c_{\mathrm{pl}}+(1-\chi) c_{\mathrm{ps}}
\end{gathered}
$$

A decoupled three-dimensional mathematic model of fluid flow and heat transfer in continuous casting billet mould was developed by An et al., (2018) [95].

$$
\frac{\partial}{\partial t}(\rho H)+\frac{\partial}{\partial x_{j}}\left(\rho \mu_{j} H\right)=\frac{\partial}{\partial x_{j}}\left[\left(\lambda+C_{p} \frac{\mu_{t}}{\sigma_{t}}\right) \frac{\partial H}{\partial x_{j}}\right]
$$

Ole Richter et al. (2017) [96] studied the development of free surface flow for the liquid and/or solid phase change. They considered enthalpy-porosity and volume-of-fluid (VOF) method.

$$
\alpha_{1}= \begin{cases}0 & =\text { gas } \\ 0<\alpha_{1}<1 & =\text { cell contains the interface } \\ 1 & =\text { solid or liquid PCM }\end{cases}
$$

The molten steel fraction was completely dependent on the thermal condition (T) of liquid metal. TS and TL indicates same respectively. This can be expressed as follows [96];

$$
\gamma_{1, l}=\left\{\begin{array}{lll}
0 & \text { if } & T<T_{S} \\
\frac{T-T_{S}}{T_{L}-T_{S}} & \text { if } & T s \leq T \leq T_{L} . \\
1 & \text { if } & T>T_{L}
\end{array}\right.
$$

Where one indicates complete liquid state and zero indicates complete solid state. In between values of solid fraction indicates mushy zone.

In the given formulation the density $\rho$, the heat capacity $\mathrm{cp}$, the heat conduction $\lambda$ and the viscosity $\mu$ can be expressed as follows;

$$
\begin{array}{r}
\rho=\alpha_{1}\left(\gamma_{1, l} \rho_{1, l}+\gamma_{1, s} \rho_{1, s}\right)+\alpha_{2} \rho_{2} \\
c_{p}=\alpha_{1}\left(\gamma_{1, l} c_{p 1, l}+\gamma_{1, s} c_{p 1, s}\right)+\alpha_{2} c_{p 2} \\
\lambda=\alpha_{1}\left(\gamma_{1, l} \lambda_{1, l}+\gamma_{1, s} \lambda_{1, s}\right)+\alpha_{2} \lambda_{2} \\
\mu=\alpha_{1} \mu_{1, l}+\alpha_{2} \mu_{2}
\end{array}
$$

In above equations, the subscripts $1,1, \mathrm{~s}$ and 2 illustatre the property of the bulk liquid, solid and gas phase, respectively. In order to consider natural convection in proposed numerical formulation, the Boussinesq approach was used. Further, the buoyancy modified density $\rho b$ can be defined as;

$$
\rho_{b}=\alpha_{1}\left(\gamma_{1, l} \rho_{1, l}\left(1-\beta\left(T-T_{L}\right)\right)+\gamma_{1, s} \rho_{1, s}\right)+\alpha_{2} \rho_{2}
$$

\section{Thermo-mechanical Deformation}

The behavior of metal especially steel at high temperature becomes sensitive to strain rate and temperature. Therefore, process design of hot metal working of steel is significantly affected by non-linear behavior of steel. Structural distortion arises in mold and strand due to thermal distribution, which causes thermal stress, cracks and ultimately affects quality strand [35, 97]. Many research has been done on mould thermal distortion in mould and strand [7, 8, 98-100]. In 2006, To measure surface temperature and shell thickness, finite point method was used by Alizadeh et al. [2]. It has been also reported heat transfer rate is affected by mold distortion [98, 101]. Many research has been done on mould thermal distortion in mould and strand [98, 99]. Generally, the heat transfer equation is solved with interfacial heat flux data and it is quantified from plant data. Subsequently, equations related to thermo-mechanical distortion in mold and strand is calculated.

$$
d \varepsilon_{i j}=d \varepsilon_{i j}^{e}+d \varepsilon_{i j}^{p}
$$

where $d \varepsilon_{i j}{ }^{e}$ and $\mathrm{d} \varepsilon_{i j}{ }^{P}$ are the incremental elastic and plastic components of the total strain vector $d \varepsilon_{i j}$

In this work they proposed incremental stresses and strains during plastic flow;

$$
d \varepsilon_{i j}^{P}=d \lambda \frac{\partial Y}{\partial \sigma_{i j}^{\prime}}
$$

where $\mathrm{d} \lambda$ is a scalar multiplying factor, $\mathrm{dY}$ is derivative of yield stress and $\sigma_{i j}$ is the deviatoric stress vector.

In 2000, Lee et al. [102] proposed a modified model of thermo-mechanical deformation in strand. They developed a mathematical model for the coupled analysis. The coupled analysis consisted of various mathematical models. The coupled model considered molten steel flow characteristics in mould. Further, it coupled the and heat transfer, thermomechanical deformation behavior of a solidifying strand in the continuous casting process. Moreover, Von-mises yield function and associated flow were assumed for increment of stress. The stress in thermo-elasto-plastic material can related as;

$$
\sigma_{i j}=C_{i j k l}\left(\varepsilon_{k l}-\varepsilon_{k l}^{P}-\varepsilon_{k l}^{T h}\right)
$$

where $C_{i j k l}, \varepsilon_{k l}, \varepsilon_{k l}^{P}$, and $\varepsilon_{k l}^{T h}$ are the elastic constitutive matrix, total infinitesimal strain, plastic strain, and thermal strain, respectively.

In a similar work, Ha et al., (2000) [79] carried a mathematical modeling for heat transfer study in secondary cooling zone of continuous casting strand. It was reported that creep was dominant factor in bulging defect. The elasticplastic creep model for the strand is given by:

$$
\dot{\varepsilon}=\alpha \sigma^{m}
$$

where $\sigma\left(\mathrm{kg} / \mathrm{cm}^{2}\right)$ and $\dot{\varepsilon}(\mathrm{I} / \mathrm{s})$ denote the equivalent stress and the creep strain rate, respectively, and $m$ is a constant of 3.15. Also 


$$
\alpha=0.0806 \exp \left\{-\frac{28392}{T+273}\right\}
$$

In 2004, Bellet et al. [8] introduced a global non-steady state (GNS) method for liquid-solid constitutive model which considered mushy zone during solidification. They reported the following relationship for total strain calculation in liquid and mushy zone;

$$
\dot{E}=\dot{\varepsilon}^{\mathrm{vp}}+\dot{\varepsilon}^{\mathrm{th}}
$$

where Evp is a strain in visco-plastic condition and Eth strain due to thermal expansion. In addition to this, a thermoelastic-viscoplastic model was used to represent the behavior in the solid state. It was described by the following equations [8];

$$
\dot{\varepsilon}=\dot{\varepsilon}^{\mathrm{el}}+\dot{\varepsilon}^{\mathrm{vp}}+\dot{\varepsilon}^{\mathrm{th}}
$$

In a similar work, Liu and Zhu (2006) [103] assumed mould copper plate should exhibit thermoelastic behavior and thermoelastic-plastic behaviour for strand. The isotropic linear elastic stress-strain relation was expressed by the constitutive equation as follows:

$$
\sigma_{i j}=2 G \varepsilon_{i j}+\left[\lambda \varepsilon_{k k}-(3 \lambda+2 G) \alpha \Delta T\right] \delta_{i j}
$$

It was reported that the total strain can be expressed as the sum of an elastic strain, a thermal strain, and a plastic strain as follows;

$$
\varepsilon_{i j}=\varepsilon_{i j}^{\mathrm{e}}+\varepsilon_{i j}^{\mathrm{p}}+\varepsilon_{i j}^{\mathrm{T}}
$$

where, temperature change $\Delta \mathrm{T}$ may induce a thermal strain of a magnitude

$$
\varepsilon_{i j}^{\mathrm{T}}=\alpha \Delta T \delta_{i j}
$$

In a recent work, Li et al. (2017) [104] reported that in the mushy zone, the stress in solid steel is supposed to increase linearly with the rise in solid fraction between zero strength temperature $\left(T_{Z S T}\right)$ at $f_{s}=0.75$ and zero Xdeformation temperature $\left(T_{Z D T}\right)$ and it can be given as follows;

$$
\sigma_{\mathrm{S}} * \frac{\left(f_{\mathrm{s}}-f_{\mathrm{ZST}}\right)}{1-f_{\mathrm{ZST}}}
$$

where $f_{s}$ is solid fraction stress, $f_{z s t}$ is stress at zero strength temperature.

Several authors have predicted the probability of crack formation in solid strand by crack susceptibility coefficient $\mathrm{SC}$ as follows [102, 105, 106];

$$
\begin{aligned}
S_{C} & =\frac{Y_{M}}{Y_{C}} \text { for }^{T} f_{S} \leq f_{S}<1 \\
& =0 \text { for } 0 \leq f_{S}<T_{S} \\
& =0 \text { for } Y_{M} \leq 0
\end{aligned}
$$

where $\varepsilon_{\mathrm{e}}$ elastics strain, $\varepsilon_{\mathrm{p}}$ Plastic Strain, $\varepsilon_{\mathrm{T}}$ Thermal strain. Thermal strain is given by

$$
\Delta\{\varepsilon\}_{\mathrm{T}}=\left(\{\alpha\}+d\left[\frac{D]_{\mathrm{c}}^{-1}\{\sigma\}}{d T}\right) d T\right.
$$

where $\alpha$ indicates coefficient of thermal expansion. Further in elastic region stress given by

$$
\Delta\{\sigma\}=[D]_{\mathrm{e}}\left(\Delta\{\varepsilon\}-\Delta\{\varepsilon\}_{\mathrm{T}}\right)
$$

where $D_{e}$ Indicates Elastic-Plastic matrix. $\sigma$ Indicates stress. Further in the plastic region the stress is given by

$$
\Delta\{\sigma\}=[D]_{\mathrm{ep}}\left(\Delta\{\varepsilon\}-\Delta\{\varepsilon\}_{\mathrm{T}}\right)+\Delta\{\sigma\}_{\mathrm{T}}
$$

Hence the thermal stress is

$$
\Delta\{\sigma\}_{\mathrm{T}}=\frac{[D]_{c}((\partial \bar{\sigma} \partial H) /(\partial\{\sigma\} \partial T)) d T}{H^{\prime}+\{\sigma \bar{\sigma} / \partial\{\sigma\}\}^{\top}\left[D_{e c}(\partial \bar{\sigma} / \partial\{\sigma\})\right.}
$$

where $\bar{\sigma}$ indicates equivalent stress at node.

They noted that near the meniscus liquid fraction is more compared to bottom slab. It shows that solidification is start early at bottom side. Because of uneven temperature in slab leads to thermal strain which creates thermal stress.

Hadata et al., [11] studied surface crack defect evaluation four criteria used namely plastic work criteria, Rice and Tracy Criteria, modified Rice and Tracy criteria and Latham criteria. Plastic work criteria can be given by following equation

$$
C_{E P}=\int_{0}^{1} \bar{\varepsilon} \bar{\sigma} d t \text { for } \sigma_{m}>0
$$

where $\varepsilon$ indicates strain rate, $\sigma$ indicates stress. This criteria based on assumption that crack will get generated if strain energy is more than critical value $C_{E P}$. Plastic strain is evaluated only in region where mean stress is positive.

Following is the criteria given by Rice \& Tracy

$$
C_{R T}=\bar{\varepsilon} \exp \left(-\frac{3}{2} \frac{6 m}{\bar{\sigma}}\right)
$$

where $\sigma_{\mathrm{m}}$ is mean stress $\& \varepsilon$ indicates strain. This criteria assumes that crack will appear if strain increases beyond $\mathrm{C}_{R T}$.

Following is the criteria given by modified which uses only positive values of strain for calculation of critical parameter $C_{R M}$. The

$$
C_{R M}=\sum \Delta \bar{\varepsilon} \exp \left(-\frac{3}{2} \frac{\sigma_{m}}{\bar{\sigma}}\right) \text { for } \sigma_{m}>0
$$

For Latham Criteria equation is as follows

$$
C_{L O}=\int_{0}^{t} \sigma_{\max } \dot{\varepsilon} \mathrm{dt} \text { for } \sigma_{m}>0
$$

\section{Conclusions}

Many literatures have reported about strand bulging between rolls which have caused transverse cracks, radial streaks and centerline macrosegregation [7, 8, 100]. Risso et al. [107] evaluated the thermal stress and strain in the solidifying shell of the strand by using the analytical method. Recently, Chen et al. (2019) [108] investigated the mold level fluctuations. These fluctuations are caused by transient bulging of the solidifying shell. Consequently, transient bulging phenomenon affects the quality of the steel. They developed a 1D and 2D model for strand 
simulation. They reported that mold level fluctuations are highly caused by dynamic bulging. Several constitutive models have been adopted for simulating the solidification stresses using the simple elastic-plastic models [109, 110]. Researchers added a separate creep model for transient modeling [111]. The integration of these transient constitutive laws and further, mathematical modeling is a challenging task. From all the above discussion it is observed that the temperature and stress-strain distribution in the strand region of the continuous casting process plays an important role in defining the quality of the final solidified product [27, 112-114]. In 2006, Liu and Zhu [103] developed a three-dimensional finite-element heat-transfer and thermal stress models to study the thermo-mechanical distortion on the slab during operation. They reported that operating parameters i.e., casting affected the strand distortion in copper walls of the mould. Pascon and coworkers (2006) [115] studied the generation of transverse crack during bending and straightening of strands. The numerical model was applied and validated with industrial data. The transverse cracks were found at the upper face of the strand. A numerical model was presented by Fachinotti et al. (2006) [100] to study the macro-segregation defects in strand caused by thermal stress. They made a hypothesis about the transient effect of alternate rolling and bulging. To measure surface temperature and shell thickness, finite point method was used by Alizadeh et al. [2]. They compared FPM results with FVM results. It was concluded that heat transfer, surface temperature, and shell thickness can be successfully modeled by FPM method.

\section{References}

[1] H. Precht and T. Preston, "Continuous Casting of Steel Slabs," in SAE Technical Paper Series, 2010, vol. 1, p. 207.

[2] M. Alizadeh, S. A. J. Jahromi, and S. B. Nasihatkon, "Applying Finite Point Method in Solidification Modeling during Continuous Casting Process," ISIJ Int., vol. 50, no. 3, pp. 411-417, 2010.

[3] D. D. Geleta, M. I. H. Siddiqui, and J. Lee, "Characterization of Slag Flow in Fixed Packed Bed of Coke Particles," Metall. Mater. Trans. B Process Metall. Mater. Process. Sci., vol. 51, no. 1, pp. 102-113, Feb. 2020.

[4] M. I. H. Siddiqui and P. K. Jha, "Numercal Investigation of Grade Intermixing and Heat Transfer during Ladle ChangeOver in Steelmaking Tundish," in 23rd International Conference on Processing and Fabrication of Advanced Materials, IIT Roorkee, 2014, pp. 981-993.

[5] M. I. H. Siddiqui and M. H. Kim, "Optimization of flow control devices to minimize the grade mixing in steelmaking tundish,” J. Mech. Sci. Technol., vol. 32, no. 7, pp. 3213-3221, 2018.

[6] M. I. H. Siddiqui and P. K. Jha, "Effect of Tundish Shape on Wall Shear Stress in a Multi-strand Steelmaking Tundish," in International Conference on Smart Technologies for Mechanical Engineering, 2013, vol. 86, no. 9999, pp. 122130.
[7] B. G. Thomas, "Modeling of the continuous casting of steelpast, present, and future," in metallurgical and materials transactions $B, 2002$, vol. 33, no. 6, pp. 3-30.

[8] M. Bellet and A. Heinrich, "A Two-dimensional Finite Element Thermomechanical Approach to a Global StressStrain Analysis of Steel Continuous Casting," ISIJ Int., vol. 44, no. 10, pp. 1686-1695, 2008.

[9] R. Kumar, M. I. H. Siddiqui, and P. K. Jha, "Numerical Investigations on the use of Flow Modifiers in Multi-Strand Continuous Casting Tundish using RTD Curves Analysis," in Proceedings of STEM-2013 International Conference on Smart Technology for Mechanical Engineers, 2013, no. October, pp. 603-612.

[10] C. Li and B. G. Thomas, "Maximum casting speed for continuous cast steel billets based on sub-mold bulging computation," 85th Steelmak. Conf. proceedings, ISS, pp. 109-130, 2002

[11] B. G. Thomas, "Issues in Thermal-Mechanical Modeling of Casting Processes.," ISIJ Int., vol. 35, no. 6, pp. 737-743, 2008.

[12] K. N. Seetharamu, R. Paragasam, G. A. Quadir, Z. A. Zainal, B. S. Prasad, and T. Sundararajan, "Finite element modelling of solidification phenomena," Sadhana-Acad. Proc. Eng. Sci., vol. 26 , no. $1-2$, pp. 103-120, Feb. 2001.

[13] M. B. N. Shaikh, M. Alam, and M. I. H. Siddiqui, "Application of Electromagnetic Forces in Continuous Casting Mold: A Review," Int. J. Adv. Prod. Mech. Eng., vol. 2, no. 5, pp. 43-49, 2016.

[14] M. Alam, T. Q. Hashmi, and M. I. H. Siddiqui, "Effect of shroud depth and advance pouring box on fluid flow and inclusion floatation behaviour in a slab caster steelmaking tundish," J. Mater. Sci. Mech. Eng., vol. 2, no. 22, pp. 19411945,2015

[15] M. I. H. Siddiqui, "Investigation of Flow Behaviour and Inclusion Removal Mechanism in a Multi-Strand Tundish With Strand Blockages," Indian Institute of Technology Roorkee, 2011.

[16] M. I. H. Siddiqui, P. K. Jha, and S. A., "Effect of Molten Steel Inflow Rate on Grade Mixing in Tundish," in National Conference on Mechanical Engineering Ideas, Innovation and Initiatives, 2016, vol. 1, no. 1, p. 221.

[17] M. I. H. Siddiqui, A. Maurya, F. Asiri, and R. Kumar, "Mathematical modeling of continuous casting tundish-A Review," VW Appl. Sci., vol. 3, no. 1, pp. 92-103, 2021.

[18] C. Li and B. G. Thomas, "Thermomechanical finite-element model of shell behavior in continuous casting of steel," Metall. Mater. Trans. B Process Metall. Mater. Process. Sci., vol. 35, no. 6, pp. 1151-1172, Dec. 2004.

[19] M. I. H. Siddiqui and P. K. Jha, "Modeling of Molten Steel Interface and Grade Mixing in a Tundish Using VOF Model," in Proceedings of the 22th National and 11th International ISHMT-ASME Heat and Mass Transfer Conference, 2013.

[20] M. I. H. Siddiqui, D. D. Geleta, G. Bae, and J. Lee, "Numerical Modeling of the Inclusion Behavior during AC Flash Butt Welding," ISIJ Int., vol. 60, no. 11, pp. 1-9, 2020 . 
[21] M. I. H. Siddiqui and M.-H. H. Kim, "Optimization of flow control devices to minimize the grade mixing in steelmaking tundish," J. Mech. Sci. Technol., vol. 32, no. 7, pp. 3213-3221, 2018.

[22] R. Kumar, A. Maurya, M. I. H. Siddiqui, and P. K. Jha, "Some studies in diffrent shapes of tundish-intermixing and flow behaviour," in 4th International Conference on Production \& Industrial Engineering, 2016.

[23] P. K. J. M. I. H. Siddiqui, “Assessment of turbulence models for prediction of intermixed amount with free surface variation using coupled level set volume of fluid method," ISIJ Int., vol. 54, no. 11, p. 2578, 2014.

[24] W. Ahmad and M. I. H. Siddiqui, "Study of Grade Intermixing and Heat Transfer in Two Different Shapes of Tundishes," in Processing and Fabrication of Advanced Materials : XXIII, 2014, vol. 2, pp. 994-1009.

[25] M. I. H. Siddiqui and P. K. Jha, "Effect of Inflow Rate Variation on Intermixing in a Steelmaking Tundish During Ladle Change-Over," Steel Res. Int., vol. 87, no. 6, pp. 733744, 2016.

[26] M. I. H. Siddiqui and M.-H. Kim, "Two-Phase Numerical Modeling of Grade Intermixing in a Steelmaking Tundish," Metals (Basel)., vol. 9, no. 1, p. 40, 2019.

[27] S. Koric, L. C. Hibbeler, and B. G. Thomas, "Explicit coupled thermo-mechanical finite element model of steel solidification," Int. J. Numer. Methods Eng., vol. 78, no. 1, pp. 1-31, Apr. 2009.

[28] Y. Yin, J. Zhang, Q. Dong, and Q. H. Zhou, "Review on Modeling and Simulation of Continuous Casting," Ironmak. Steelmak., vol. 46, no. 9, pp. 855-864, 2019.

[29] X. Huang and B. G. Thomas, "Intermixing model of continuous casting during a grade transition," Metall. Mater. Trans. B, vol. 27, no. 4, pp. 617-632, Apr. 1996.

[30] B. Petrus, K. Zheng, X. Zhou, B. G. Thomas, and J. Bentsman, "Real-time, model-based spray-cooling control system for steel continuous casting," Metall. Mater. Trans. B Process Metall. Mater. Process. Sci., vol. 42, no. 1, pp. 87103, Feb. 2011.

[31] B. G. Thomas and L. Zhang, "Mathematical Modeling of Fluid Flow in Continuous Casting," Rev. Lit. Arts Am., vol. 41, no. 10, pp. 1181-1193, 2001.

[32] Y. Meng and B. G. Thomas, "Modeling Transient Slag-Layer Phenomena in the Shell/mold Gap in Continuous Casting of Steel," Metall. Mater. Trans. B Process Metall. Mater. Process. Sci., vol. 34, no. 5, pp. 707-725, 2003.

[33] J. Mahmoudi, "Mathematical modelling of fluid flow, heat transfer and solidification in a strip continuous casting process," Int. J. Cast Met. Res., vol. 19, no. 4, pp. 223-236, 2006.

[34] Z.-D. Qian and Y.-L. Wu, "Large Eddy Simulation of Turbulent Flow with the Effects of DC Magnetic Field and Vortex Brake Application in Continuous Casting," ISIJ Int., vol. 44, no. 1, pp. 100-107, 2004.

[35] J. K. Park, B. G. Thomas, I. V. Samarasekera, and S. U. Yoon, "Thermal and mechanical behavior of copper molds during thin-slab casting (I): Plant trial and mathematical modeling," Metall. Mater. Trans. B Process Metall. Mater. Process. Sci., vol. 33, no. 3, pp. 425-436, 2002.
[36] S. Mazumdar and S. K. Ray, "Solidification control in continuous casting of steel," Sadhana, vol. 26, no. 1-2, pp. 179-198, 2001.

[37] B. Hadała, A. Cebo-Rudnicka, Z. Malinowski, and A. Gołdasz, "The Influence of Thermal Stresses and Strand Bending on Surface Defects Formation in Continuously Cast Strands," Arch. Metall. Mater, vol. 56, no. 2, pp. 367-377, 2011.

[38] M. R. R. I. Shamsi and S. K. Ajmani, "Three Dimensional Turbulent Fluid Flow and Heat Transfer Mathematical Model for the Analysis of a Continuous Slab Caster," ISIJ Int., vol. 47, no. 3, pp. 433-442, 2007.

[39] Y. Sahai, "Tundish Technology for Casting Clean Steel: A Review," Metall. Mater. Trans. B Process Metall. Mater. Process. Sci., vol. 47, no. 4, pp. 2095-2106, 2016.

[40] A. E. Huespe, A. Cardona, and V. Fachinotti, "Thermomechanical model of a continuous casting process," Comput. Methods Appl. Mech. Eng., vol. 182, no. 3-4, pp. 439-455, 2000.

[41] J. X. Fu, W. S. Hwang, J. S. Li, S. F. Yang, and Z. Hui, "Effect of casting speed on slab broadening in continuous casting," Steel Res. Int., vol. 82, no. 11, pp. 1266-1272, 2011.

[42] J. R. Boehmer, G. Funk, M. Jordan, and F. N. Fett, "Strategies for coupled analysis of thermal strain history during continuous solidification processes," Adv. Eng. Softw., vol. 29, no. 7-9, pp. 679-697, Aug. 1998.

[43] J. Svensson, P. E. R. López, P. N. Jalali, and M. Cervantes, "One-way coupling of an advanced CFD multi-physics model to FEA for predicting stress-strain in the solidifying shell during continuous casting of steel," IOP Conf. Ser. Mater. Sci. Eng., vol. 84, no. 1, 2015.

[44] B.-Z. Ren, D.-F. Chen, H.-D. Wang, M.-J. Long, and Z.-W. Han, "Numerical simulation of fluid flow and solidification in bloom continuous casting mould with electromagnetic stirring," Ironmak. Steelmak., vol. 42, no. 6, pp. 401-408, 2015.

[45] X. Jin*, D. F. Chen, D. J. Zhang, X. Xie, Y. Y. Bi, and X. Jin, "Water model study on fluid flow in slab continuous casting mould with solidified shell," Ironmak. Steelmak., vol. 38, no. 2, pp. 155-159, Feb. 2011.

[46] H. K. Versteeg and W. Malalasekera, An Introduction to Computational Fluid Dynamics: The Finite Volume Method, 2nd-ed ed. Pearson, Prentice Hall, 2007.

[47] B. Zhao, B. G. Thomas, S. P. Vanka, and R. J. O'Malley, "Transient Flow and Temperature Transport in Continuous Casting of Steel Slabs," J. Heat Transfer, vol. 127, no. 8, p. $807,2005$.

[48] B. Li and F. Tsukihashi, "Vortexing Flow Patterns in a Water Model of Slab Continuous Casting Mold," ISIJ Int., vol. 45, no. 1, pp. 30-36, 2005.

[49] L. Sowa and A. Bokota, "Numerical model of thermal and flow phenomena the process growing of the CC slab," Arch. Metall. Mater, vol. 56, no. 2, pp. 359-366, 2011.

[50] M. H. H. Zare, a. H. H. Meysami, S. Mahmoudi, M. Hajisafari, and M. Mazar Atabaki, "Simulation of flow field and steel/slag interface in the mold region of a thin slab steel continuous caster with tetra-furcated nozzle," J. Manuf. Process., vol. 15, no. 3, pp. 307-317, Aug. 2013. 
[51] W. Chen, Y. Ren, L. Zhang, and P. R. Scheller, "Numerical Simulation of Steel and Argon Gas Two-Phase Flow in Continuous Casting Using LES + VOF + DPM Model," Jom, vol. 71, no. 3, pp. 1158-1168, 2019.

[52] M. I. H. Siddiqui, Ambrish Maurya, Rajneesh Kumar, "Advancements in numerical modeling of the continuous casting mold," VW Engineering International., vol. 3, no. 1, pp. 1-22, 2021.

[53] M. I. H. Siddiqui and M. H. Kim, "Two-phase numerical modeling of grade intermixing in a steelmaking Tundish," Metals (Basel)., vol. 9, no. 1, p. 40, Jan. 2019.

[54] J. R. De Sousa Rocha, E. E. B. De Souza, F. Marcondes, and J. A. De Castro, "Modeling and computational simulation of fluid flow, heat transfer and inclusions trajectories in a tundish of a steel continuous casting machine," J. Mater. Res. Technol., vol. 8, no. 5, pp. 4209-4220, Sep. 2019.

[55] D.-Y. Sheng and Q. Yue, "Modeling of Fluid Flow and Residence-Time Distribution in a Five-Strand Tundish," Met. 2020, Vol. 10, Page 1084, vol. 10, no. 8, p. 1084, Aug. 2020.

[56] M. I. H. Siddiqui, Ambrish Maurya, Rajneesh Kumar, "Advancements in numerical modeling of the continuous casting mold," VW Engineering International, vol. 3, no. 1, pp. $1-22,2021$.

[57] M. I. H. Siddiqui, H. Alshehri, J. Orfi, M. A. Ali, and D. Dobrota, "Computational Fluid Dynamics (CFD) Simulation of Inclusion Motion under Interfacial Tension in a Flash Welding Process," Metals (Basel)., vol. 11, no. 7, p. 1073, 2021.

[58] Y. Ruan, Y. Yao, S. Shen, B. Wang, J. Zhang, and J. Huang, "Physical and Mathematical Simulation of Surface-Free Vortex Formation and Vortex Prevention Design during the End of Casting in Tundish," steel Res. Int., vol. 91, no. 6, p. 1900616, Jun. 2020.

[59] M. A. Saeidy Pour and S. Hassanpour, "Steel Cleanliness Depends on Inflow Turbulence Intensity (in Tundishes and Molds)," Metall. Mater. Trans. B Process Metall. Mater. Process. Sci., vol. 51, no. 5, pp. 2199-2210, Oct. 2020.

[60] M. I. H. Siddiqui and P. K. Jha, "Assessment of Turbulence Models for Prediction of Intermixed Amount with Free Surface Variation Using Coupled Level-Set Volume of Fluid Method," ISIJ Int., vol. 54, no. 11, pp. 2578-2587, 2014.

[61] Y. Liu, Z. He, and L. Pan, "Numerical Investigations on the Slag Eye in Steel Ladles," Adv. Mech. Eng., vol. 2014, pp. 1-6, 2014.

[62] H.-X. Li, Q. Wang, H. Lei, J.-W. Jiang, Z.-C. Guo, and J.-C. He, "Mechanism Analysis of Free-Surface Vortex Formation during Steel Teeming," ISIJ Int., vol. 54, no. 7, pp. 1592-1600, 2014.

[63] J. Szekely and R. T. Yadoya, "The physical and mathematical modelling of the flow field in the mold region in continuous casting systems: Part II. The mathematical representation of the turbulent flow field," Metall. Trans., vol. 4, no. 5, pp. 1379-1388, 1973.

[64] S. K. Choudhary and D. Mazumdar, "Mathematical modelling of fluid flow, heat transfer and solidification phenomena in continuous casting of steel," Steel Res., vol. 66, no. 5, pp. 199-205, May 1995.

[65] ANSYS FLUENT Theory Guide, 18.2., no. August. Canonsburg, PA: ANSYS Inc. USA, 2017.
[66] M. I. H. Siddiqui and P. K. Jha, "Numerical Analysis of Heat Transfer and Flow Behaviour inside Different Shapes of Multi-Strand Continuous Casting Tundish," in 2nd National Conference on Advances in Heat Transfer and Fluid Dynamics, AMU, Aligarh, India, 2013, pp. 65-72.

[67] M. Alam and M. I. H. Siddiqui, "CFD simulation of melt and inclusion motion in a mold under the influence of electromagnetic force," VW Appl. Sci., vol. 1, no. 1, pp. 7-14, 2019.

[68] M. I. H. Siddiqui and P. K. Jha, "Multi-phase analysis of steelair-slag system during ladle change-over process in CC tundish steelmaking process," in Asia Steel Conference.

[69] M. I. H. Siddiqui et al., "Physical Investigations of Grade Mixing Phenomenon in Delta Shape Steel-making Tundish," Int. Conf. CETCME, NIET, Noida, India, vol. 2, no. 13, pp. 94-98, 2015.

[70] M. V. More, S. K. Saha, V. Marje, and G. Balachandran, "Numerical model of liquid metal flow in steel making tundish with flow modifiers," IOP Conf. Ser. Mater. Sci. Eng., vol. 191, no. 1, 2017.

[71] R. Chaudhary, C. Ji, B. G. Thomas, and S. P. Vanka, "Transient turbulent flow in a liquid-metal model of continuous casting, including comparison of six different methods," Metall. Mater. Trans. B Process Metall. Mater. Process. Sci., vol. 42, no. 5, pp. 987-1007, 2011.

[72] L. C. Hibbeler, R. Liu, and B. G. Thomas, "Review of Mold Flux Entrainment Mechanisms and Model Investigation of Entrainment by Shear-Layer Instability Meniscus Freezing and Hook Formation Another mechanism for the entrainment of slag and," InSteelCon, no. July, pp. 1-10, 2011.

[73] C. Kratzsch, K. Timmel, S. Eckert, and R. Schwarze, "URANS Simulation of Continuous Casting Mold Flow: Assessment of Revised Turbulence Models," steel Res. Int., vol. 86, no. 4, pp. 400-410, Apr. 2015.

[74] L. C. Hibbeler and B. G. Thomas, "Mold slag entrainment mechanisms in continuous casting molds," Iron Steel Technol., vol. 10, no. 10, pp. 121-136, 2013.

[75] W. Chen, Y. Ren, and L. Zhang, "Large Eddy Simulation on the Two-Phase Flow in a Water Model of Continuous Casting Strand with Gas Injection," Steel Res. Int., vol. 1800287, pp. $1-12,2018$.

[76] T. Vu, C. Nguyen, and D. Khanh, "Direct Numerical Study of a Molten Metal Drop Solidifying on a Cold Plate with Different Wettability," Metals (Basel)., vol. 8, no. 1, p. 47, 2018.

[77] B. G. Thomas, Q. Yuan, S. Sivaramakrishnan, T. Shi, S. P. Vanka, and M. B. Assar, "Mathematical Modeling of Iron and Steel Making Processes. Comparison of Four Methods to Evaluate Fluid Velocities in a Continuous Slab Casting Mold.," ISIJ Int., vol. 41, no. 10, pp. 1262-1271, 2008.

[78] B. Launder and D. Spalding, "The Numerical Computation of Turbulent Flows," Comput. Methods Appl. Mech. Eng., vol. 3, pp. 269-289, 1974.

[79] J. S. Ha, J. R. Cho, B. Y. Lee, and M. Y. Ha, "Numerical analysis of secondary cooling and bulging in the continuous casting of slabs," J. Mater. Process. Technol., vol. 113, no. 13, pp. 257-261, 2001. 
[80] J. R. Boehmer, F. N. Fett, and G. Funk, "Analysis of hightemperature behaviour of solidified material within a continuous casting machine," Comput. Struct., vol. 47, no. 4-5, pp. 683-698, 1993.

[81] K. Härkki and J. Miettinen, "Mathematical modeling of copper and brass upcasting," Metall. Mater. Trans. B, vol. 30, no. 1, pp. 75-98, Feb. 1999.

[82] M. L. S. Zappulla, L. C. Hibbeler, and B. G. Thomas, "Effect of Grade on Thermal-Mechanical Behavior of Steel During Initial Solidification," Metall. Mater. Trans. A Phys. Metall. Mater. Sci., vol. 48, no. 8, pp. 3777-3793, Aug. 2017.

[83] A. M, E. H, and S. A., "Mathematical modeling of heat transfer for steel continuous casting process," Int J ISSI, vol. 3 , no. 2, pp. 7-16, 2006.

[84] C. A. M. Pinheiro, I. V. Samarasekera, J. K. Brimacomb, and B. N. Walker, "Mould heat transfer and continuously cast billet quality with mould flux lubrication Part 1 Mould heat transfer," Ironmak. Steelmak., vol. 27, no. 1, pp. 37-54, Feb. 2003.

[85] Q. Wang, Z. He, B. Li, and F. Tsukihashi, "A General Coupled Mathematical Model of Electromagnetic Phenomena, Two-Phase Flow, and Heat Transfer in Electroslag Remelting Process Including Conducting in the Mold," Metall. Mater. Trans. B Process Metall. Mater. Process. Sci., vol. 45, no. 6, pp. 2425-2441, 2014.

[86] A. C. Kheirabadi and D. Groulx, "the Effect of the MushyZone Constant on Simulated Phase Change Heat Transfer," Proceeding Proc. CHT-15. 6th Int. Symp. Adv. Comput. HEAT Transf., May 25-29, 2015, Rutgers Univ. New Brunswick, NJ, USA, no. May, p. 22, 2015.

[87] A. Maurya and P. K. Jha, "Influence of electromagnetic stirrer position on fluid flow and solidification in continuous casting mold," Appl. Math. Model., vol. 48, pp. 736-748, 2017.

[88] S. Louhenkilpi, M. Mäkinen, S. Vapalahti, T. Räisänen, and J. Laine, "3D steady state and transient simulation tools for heat transfer and solidification in continuous casting," Mater. Sci. Eng. A, vol. 413-414, pp. 135-138, 2005.

[89] A. A. Ivanova, "Calculation of Phase-Change Boundary Position in Continuous Casting," Arch. Foundry Eng., vol. 13, no. 4, pp. 57-62, 2013.

[90] D. Zhang, S. Lei, S. Zeng, and H. Shen, "Thermo-mechanical Modeling in Continuous Slab Casting Mould and Its Application," ISIJ Int., vol. 54, no. 2, pp. 336-341, 2014.

[91] Ambrish Maurya and Pradeep Kumar Jha, "Effect of Casting Speed on Continuous Casting of Steel Slab," Int. J. Mech. Eng. Robot. Res., vol. 1, no. 1, pp. 13-21, 2014.

[92] P. T. Hietanen, S. Louhenkilpi, and S. Yu, "Investigation of Solidification, Heat Transfer and Fluid Flow in Continuous Casting of Steel Using an Advanced Modeling Approach," Steel Res. Int., vol. 88, no. 7, pp. 1-13, 2017.

[93] L. C. Hibbeler, M. M. Chin See, J. Iwasaki, K. E. Swartz, R. J. O'Malley, and B. G. Thomas, "A reduced-order model of mould heat transfer in the continuous casting of steel," Appl. Math. Model., vol. 40, no. 19-20, pp. 8530-8551, 2016.

[94] M. Vynnycky and S. Saleem, "On the explicit resolution of the mushy zone in the modelling of the continuous casting of alloys," Appl. Math. Model., vol. 50, pp. 544-568, 2017.
[95] Y. Yin, J. Zhang, Q. Dong, and Q. H. Zhou, "Effects of electromagnetic stirring on fluid flow and temperature distribution in billet continuous casting mould and solidification structure of 55SiCr," Ironmak. Steelmak., vol. 46, no. 9, pp. 855-864, 2019.

[96] O. Richter, J. Turnow, N. Kornev, and E. Hassel, "Numerical simulation of casting processes: coupled mould filling and solidification using VOF and enthalpy-porosity method," Heat Mass Transf. und Stoffuebertragung, vol. 53, no. 6, pp. 19571969, 2017.

[97] T. Oconnor and D. JA, "Modeling the thin-slab continuouscasting mold," Metall. Mater. Trans. B, vol. 25B, no. 6, p. 443, 1994.

[98] I. V. Samarasekera, D. L. Anderson, and J. K. Brimacombe, "The thermal distortion of continuous-casting billet molds," Metall. Trans. B, vol. 13, no. 1, pp. 91-104, 1982.

[99] H. A. N. K. Hwan, J. Yoon, and J. L. E. E. H. Nam, "Coupled Analysis of Fluid Flow, Heat Transfer and Stress Continuous Round Billet Oasting Thermal Analysis in Mold," ISIJ Int., vol. 39, no. 5, pp. 435-444, 1999.

[100] V. D. Fachinotti, S. Le Corre, N. Triolet, M. Bobadilla, and M. Bellet, "Two-phase thermo-mechanical and macrosegregation modelling of binary alloys solidification with emphasis on the secondary cooling stage of steel slab continuous casting processes," Int. J. Numer. Methods Eng., vol. 67, no. 10, pp. 1341-1384, Sep. 2006.

[101] Y. Hebi, Y. Man, Z. Huiying, and F. Dacheng, "3D Stress Model with Friction in and of Mould for Round Billet Continuous Casting," ISIJ Int., vol. 46, no. 4, pp. 546-552, 2006.

[102] J. E. Lee, T. J. Yeo, O. H. Kyu Hwan, J. K. Yoon, and U. S. Yoon, "Prediction of cracks in continuously cast steel beam blank through fully coupled analysis of fluid flow, heat transfer, and deformation behavior of a solidifying shell," Metall. Mater. Trans. A Phys. Metall. Mater. Sci., vol. 31, no. 1, pp. 225-237, 2000.

[103] X. Liu and M. Zhu, "Finite Element Analysis of Thermal and Mechanical Behavior in a Slab Continuous Casting Mold," ISIJ Int., vol. 46, no. 11, pp. 1652-1659, 2006.

[104] Y. jun Li, H. Li, P. Lan, H. yan Tang, and J. quan Zhang, "Thermo-elasto-visco-plastic finite element analysis on formation and propagation of of-corner subsurface cracks in bloom continuous casting," J. Iron Steel Res. Int., vol. 24, no. 11, pp. 1159-1168, 2017.

[105] M. R. Ridolfi, "Hot tearing modeling: A microstructural approach applied to steel solidification," Metall. Mater. Trans. B Process Metall. Mater. Process. Sci., vol. 45, no. 4, pp. 1425-1438, 2014.

[106] S. Saleem, M. Vynnycky, and H. Fredriksson, "The Influence of Peritectic Reaction/Transformation on Crack Susceptibility in the Continuous Casting of Steels," Metall. Mater. Trans. B Process Metall. Mater. Process. Sci., vol. 48, no. 3, pp. 1625 1635, 2017.

[107] J. M. Risso, A. E. Huespe, and A. Cardona, "Thermal stress evaluation in the steel continuous casting process," Int. J. Numer. Methods Eng., vol. 65, no. 9, pp. 1355-1377, 2006.

[108] Z. Chen, H. Olia, B. Petrus, M. Rembold, J. Bentsman, and B. G. Thomas, "Dynamic Modeling of Unsteady Bulging in Continuous Casting of Steel," in Materials Processing Fundamentals, 2019, pp. 23-35. 
[109] A. G. Weinberg, Brimacombe, and J. K. F., "Mathematical analysis of stress in continuous casting of steel," Ironmak. Steelmak., vol. 3, no. 1, pp. 38-47, 1976.

[110] J. E. Kelly, K. P. Michalek, T. G. O'Connor, B. G. Thomas, and J. A. Dantzig, "Initial development of thermal and stress fields in continuously cast steel billets," Metall. Trans. A, Phys. Metall. Mater. Sci., vol. 19 A, no. 10, pp. 2589-2602, Oct. 1988.

[111] J. O. Kristiansson, "Thermomechanical behavior of the solidifying shell within continuous-casting billet molds-a numerical approach," J. Therm. Stress., vol. 7, no. 3-4, pp. 209-226, Jan. 1984.

[112] M. Y. Zhu, Z. Z. Cai, and H. Q. Yu, "Multiphase Flow and
Thermo-Mechanical Behaviors of Solidifying Shell in Continuous Casting Mold," J. Iron Steel Res. Int., vol. 20, no. 3, pp. 6-17, 2013.

[113] G. Funk, J. R. Boehmer, and F. N. Fett, "A coupled FDM/FEM model for the continuous casting process," Int. $J$. Comput. Appl. Technol., vol. 7, no. 3-6, 1994.

[114] M. Samonds and J. Z. Zhu, "Coupled Thermal-fluids-stress Analysis of Castings," in Proc. 9 th Int. Conf. on Modeling of Casting, 2000.

[115] F. Pascon, S. Cescotto, and A. M. Habraken, "A 2.5D finite element model for bending and straightening in continuous casting of steel slabs," Int. J. Numer. Methods Eng., vol. 68, no. 1, pp. 125-149, Oct. 2006. 